\title{
CHARACTERIZATION BY ATOMIC FORCE MICROSCOPY AND CRYOELECTRON MICROSCOPY OF TAU POLYMERS ASSEMBLED IN ALZHEIMER'S DISEASE
}

Fernando Moreno-Herrero ${ }^{1}$, José M. Valpuesta ${ }^{3}$, Mar Pérez $^{2}$, Jaime Colchero ${ }^{1}$, Arturo M. Baró ${ }^{1}$, Jesús Avila $^{2}$, Esteban Montejo de Garcini ${ }^{2}$

${ }^{1}$ Departamento de Física de la Materia Condensada. Facultad de Ciencias. Universidad Autónoma de Madrid. 28049 Madrid. Spain.

2 Centro de Biología Molecular "Severo Ochoa" (UAM-CSIC). Facultad de Ciencias. Universidad Autónoma de Madrid. 28049 Madrid. Spain.

${ }^{3}$ Centro Nacional de Biotecnología (CSIC). Campus de la Universidad Autónoma de Madrid. 28049 Madrid. Spain.

This work was supported by a grant of Comunidad Autónoma de Madrid (to A.M.B.) and by grants from CICYT (Spain) and Fundación La Caixa

Keywords: tau protein / assembly / neurodegeneration /Alzheimer's disease /Atomic Force Microscopy / Cryoelectron microscopy

short title: The ribbon nature of PHF polymers.

*Corresponding author and reprint request

Jesús Avila

Centro de Biología Molecular "Severo Ochoa"

Fac. Ciencias, Universidad Autónoma de Madrid, Cantoblanco, 28049 Madrid, Spain

Telephone: 34-91-3978440

Fax: 34-91-3974799

E-mail: javila@cbm.uam.es 


\begin{abstract}
The structure of the Paired Helical filaments $(\mathrm{PHF})^{1}$, a polymer of the microtubule associated protein tau, has been studied by Atomic Force Microscopy (AFM) and by cryoelectron microscopy. Mica and graphite were used as substrates in the AFM analysis with no differences in the results. A banding pattern of 8-12 nm width within the helical structure is found when detailed analysis of the data is performed. High AFM resolution images obtained by using an ultra sharp tip confirm the previous results and suggest that the structures observed are compatible with a helical ribbon made up of two parallel strands. These results were confirmed by cryoelectron microscopy experiments.
\end{abstract}

\footnotetext{
${ }^{1}$ Abbreviations: AFM: Atomic Force Microscopy; NFT: Neurofibrillay tangles; AD: Alzheimer's Disease; PHF: Paired Helical Filaments. SP: Senile Plaques.
} 


\section{INTRODUCTION}

Alzheimer's disease (AD) is a dementia showing some pathological features that have been related with the onset of progressive cognitive impairments. Two major aberrant structures are found in the brain of $\mathrm{AD}$ patients, the extracellular senile plaques (SP) and the intracellular (after cell death could become extracellular) neurofibrillary tangles (NFT) [10,11,29]. Since the presence of NFT is closely correlated with the degree of dementia in AD patients [2], the study of these structures has deserved an important attention. NFTs have been found to be aggregates of filamentous polymers termed paired helical filaments (PHF) [19], although Terry [42] described the components of NFT as ribbon-like twisted tubules. Biochemical studies have indicated that the main component of PHF is the microtubule-associated protein termed tau $[4,14,17,20,21,30,49,50,52]$. PHF-like polymers could be assembled in vitro using tau $[7,24,25,45]$, being a short region of this protein the minimum sequence required for its selfassembly [32]. The structural characterization of PHF has improved during the last years thanks to the use of techniques such as X-ray diffraction [51] and electron microscopy $[1,24,31,39,47]$.

The studies by Ruben et al. $[36,38]$ have lead to suggest that PHF could be made of non-periodic thin helical ribbons. However, this point has been challenged [18] and it has even been suggested that the real structure of PHF could be a hybrid of the two, the paired helical filament model and that of the thin helical ribbon model [34]. AFM is a very adequate technique to observe biomolecules and other molecular assemblies [27,28,43]. From the structural point of view, the technique can image structures in native conditions, in three dimensions without staining or shadowing, in air or under liquid conditions (see for reviews [5,8,40]). Atomic Force Microscopy (AFM) has already been used to confirm the helical nature of the PHFs [33]; see also [35]. Cryoelectron microscopy, a technique capable of extracting three-dimensional information out of the specimen, can be used to complement the information on the surface generated by the AFM. 
In this work, both complementary techniques have been used to further characterize the nature of PHFs. 


\section{MATERIALS AND METHODS}

The tissues used in this work were obtained from the Netherlands Brain Bank and from the Cambridge Brain Bank. Brains were collected from autopsies (less than 18 hours postmortem) from four patients with clinically confirmed Alzheimer's disease.

\section{$\underline{\text { PHF isolation }}$}

PHF fractions were obtained as described using the method of [13] PHF protein was subjected to SDS acrylamide gel electrophoresis and the fractionated protein was characterized by Western blot using PHF-1 antibody (a kind gift of Dr. P. Davies, Albert Einstein University, Bronx, NY). Additionally, the presence of PHF was determined by electron microscopy as previously described [30].

Tau was isolated from bovine brain microtubules by following the procedure previously indicated.

\section{$\underline{\text { AFM sample preparation }}$}

PHF samples were prepared as follows: $4 \mu$ of the PHFs sample were deposited on freshly cleaved mica and allowed to bind for one minute. Preparations were then rinsed with 3-5 ml MilliQ water and the excess liquid wicked away at the mica edge with a tissue paper. The mica was then blown completed dry with a stream of nitrogen gas. Samples prepared this way were immediately imaged with the microscope.

\section{$\underline{\text { AFM imaging }}$}

AFM uses a sharp tip at the end of a flexible cantilever to scan and sense the topography of a sample deposited on a flat surface [3], usually mica or glass. The AFM images were obtained with

a commercial microscope (Nanotec Electronica S.L., Spain) operating in non contact tapping 
mode following the conditions described in detail elsewhere [26], for the Olympus type cantilevers employed $(15 \mathrm{~nm}$ nominal radius, $\mathrm{k}=1 \mathrm{~N} / \mathrm{m}$, resonance frequency about $80 \mathrm{kHz}$ ). To image the surface in noncontact AFM the tip is oscillated at its free resonance frequency and the normal force signal is processed to measure the amplitude and the relative phase of the oscillation. Our digital control electronics then establishes feedback at a certain oscillation amplitude which is

slightly lower than the free resonance amplitude. Two types of cantilevers were used: Olympus type (see above) and SuperSharpSilicon ${ }^{\circledR}$-Tips (Nanosensors $\left.{ }^{\circledR}\right)$ with a nominal radius equal or less than $5 \mathrm{~nm}$, a force constant of $10 \mathrm{~N} / \mathrm{m}$, and a resonance frequency of $120 \mathrm{kHz}$. Images were recorded at typical scan frequencies of $2-3 \mathrm{~Hz}$ and the set point was chosen as high as possible to keep tip-sample interaction the softest. Images were collected in air at room temperature at approx. $30 \%$ relative humidity. Some images were processed by subtracting a general plane to remove the background slope and filtered to eliminate the low frequency noise.

\section{$\underline{\text { Mathematical modeling and dilation simulation }}$}

AFM images of biological structures are characterized for an overestimated lateral dimension because the typical lateral size of a molecule is smaller than the lateral size of the tip (tip radius) [5]. In this way a dilation phenomenon is often present in AFM imaging of biological assemblies. To determine whether AFM images are compatible with either an ideal two-strand structure or an ideal thin twisted ribbon, different models were generated. A dilation simulation was carried out as described in [44], where the tip-sample interaction is modeled like contact without penetration of the tip into the sample. More than a thousand simulations were carried out searching for the best fit of the tip parameters employed for the dilation. 


\section{Cryoelectron microscopy}

Grids were prepared by evaporating carbon onto freshly cleaved mica, which was then floated on water and lowered on to the grids. These were glow-discharged in air. $3 \mu$ l aliquots of PHF samples prepared as described above were applied to the carbon film for $3 \mathrm{~min}$, blotted for $5 \mathrm{~s}$ and quickly frozen in liquid ethane at $-180{ }^{\circ} \mathrm{C}$, so that the specimen was preserved in vitreous ice. The frozen grids were kept under liquid nitrogen until used. Electron micrographs were recorded on Kodak SO-163 film using low electron dose conditions with a JEOL 1200EX-II operated at 100 $\mathrm{kV}$. The images were obtained with a nominal magnification of $\mathrm{x} 50000$ and a nominal underfocus

varying from 10000 to $20000 \AA$. A GATAN-626 cold stage was used in the recording of the electron micrographs from specimens at temperatures below $-160{ }^{\circ} \mathrm{C}$. 


\section{RESULTS}

\section{AFM analysis of the PHF polymers}

PHF were prepared as described above and analyzed by AFM. All experiments have given similar results. Fig.1A and Fig.1B show a typical set of PHF particles at different magnifications. From these images, polymers with helical profiles and dimensions similar to those obtained by other authors [46] can be observed. The average pitch calculated for PHFs from different preparations is $82 \mathrm{~nm}$, with a height of $8 \mathrm{~nm}$ in the "valley" and with a height of $16 \mathrm{~nm}$ in the "top" of the helical structure.

Previous analyses have been performed using mica as substrate [34]. However, in a recent work by AFM [22], it has been suggested that for the analysis of other similar polymers like amyloid fibers, graphite seems to be a more suitable substrate. The same type of experiments were then carried out with graphite, which gave similar results to those obtained with mica. A detailed analysis of the images obtained reveals, regardless of the support used, a banding pattern of 8-12 nm width within the helical structure. Fig. 1C shows the derivative image of a PHF particle. These data support the idea of a basic model of a helix being constituted by a basic structural element repeated periodically. The three-dimensional representation of the PHF shown in Fig. 1D reinforces the presence of this repetitive structure that has not been described previously. This structure could be formed by several molecules of tau.

\section{$\underline{\text { AFM analysis using a ultra-sharp tip }}$}

Looking for a higher resolution, a tip with a nominal diameter equal or smaller than $5 \mathrm{~nm}$ was used in the subsequent AFM analyses (Fig. 2). Again, the helix structure was observed as basic structural element, but more information about the polymer structure was obtained. Fig. 2A and B 
show two of the filaments imaged at high magnification. Unlike in the previous observations where PHFs seemed to be formed by a thick helical structures, here the filament seems to be generated by a thin twisted ribbon with a $82 \mathrm{~nm}$ pitch (Fig. 2C). Assuming a tip radius of $5 \mathrm{~nm}$, and the measured values of the thickness and width (Fig 2D and Fig. 2E), we can calculate, by geometrical arguments as the ones pointed in [23], the thickness and the width of the fiber. And average thickness and width of 3 and $16 \mathrm{~nm}$ is calculated. The blurred image obtained in the "valleys" of the filaments can be explained by the tip deforming this part of the structure that, because of its helical nature, is hanging between the domains interacting with the support material. A more careful observation of the width profile (Fig. 2E) allows detecting a two-peak pattern, suggesting that a two-strand ribbon (see discussion) may form the helix. Similar results were obtained with different PHF preparations (not shown).

An interesting point in the images taken with the supertips is the absence of the banded pattern. As will be discussed in more detail below, this might be related to differences in the interaction of the two kind of tips used, since they are not chemically identical.

\section{Cryoelectron microscopy analysis}

To confirm the existence of the ribbon structures we have used a different technique, cryoelectron microscopy, in which the PHF polymers are quickly frozen in the physiological buffer in which they are suspended, and thus their native structure is preserved. Fig. 3 shows several examples of such filaments. In all of them, a similar architecture as that obtained by AFM with the $5 \mathrm{~nm}$ tip is observed: a thin ribbon of $16 \mathrm{~nm}$ width and $3 \mathrm{~nm}$ thickness turning around its longitudinal axis generating the classical helix which has defined these filaments. In some of them (see arrow in

Fig. 3A), a periodic substructure of average $8 \mathrm{~nm}$ similar to that detected by AFM could be observed perpendicular to the longitudinal axis. The data support the idea that the helical ribbon is 
formed by rectangular units of $8 \mathrm{~nm} \times 16 \mathrm{~nm}$ placed perpendicular to the longitudinal axis of the helix.

\section{$\underline{\text { AFM simulation }}$}

To further test that the PHFs are indeed twisted ribbons and try to characterize the nature of this these ribbons (whether they were made up by one or two strands), a simulation of the results obtained by AFM was carried out and subsequently compared with the experimental results (Fig. 4). Three different models were used in the simulation. Model 1 is a twisted ribbon of $16 \mathrm{~nm}$ width (Fig. 4C), model 2 consists of two paired strands of $4 \mathrm{~nm}$ thickness separated by $9 \mathrm{~nm}$ (Fig. 4F), and model 3 consists of two strands of $8 \mathrm{~nm}$ wide separated by $1 \mathrm{~nm}$ (Fig. 4I), which is approximately the width of the cleft found in the lateral profile of the PHFs imaged by the ultrasharp tip (Fig. 2E). The three theoretical models were simulated to be scanned with either the standard $15 \mathrm{~nm}$ tip (Fig. 4E, H and K) or the $5 \mathrm{~nm}$ tip (Fig. 4D, G and J). All the simulation generated can be compared with images of PHFs obtained experimentally with the $5 \mathrm{~nm}$ tip (Fig. 4B) or the standard tip (Fig. 4C). The results of the simulation reveal clearly that none of the proposed models could be resolved using the conventional tip since the three simulations generated are essentially identical. However, simulations with the three models and the ultra-sharp tip reveal differences between the three images generated. Whereas it is clear that the image obtained using the second model (Fig. 4G) is not compatible with the experimental image (Fig. 4A), the images generated using the first model (one strand occupying the whole ribbon; Fig. 4D) or the third model (a two-strand ribbon with a small separation between them; Fig. 4J) could fit with the experimental image. 


\section{DISCUSSION}

The structural analyses carried out in this work with two different and complementary techniques, AFM and cryoelectron microscopy, have first confirmed that the paired helical filaments are indeed formed by a helical structure, as it has been already described $[1,34,39,47,48]$. The AFM analysis was performed with two types of supports, mica and graphite, which have different hydrophobicity properties. However, no main differences between the two supports were observed and similar results to those previously described were found [34]. A periodical substructure is found when further analyses are performed. (Fig. 1C and D). The existence of this substructure is confirmed by cryoelectron microscopy analysis (Fig. 3A) in which the same rectangular structures of approximately $8 \mathrm{~nm}$ wide $\times 16 \mathrm{~nm}$ long are observed.

A dramatic change in resolution is obtained when using a very sharp tip $(5 \mathrm{~nm})$ in which the dilation phenomena is minimized. The images generated by this tip (Fig. 2) reveal that the PHF is composed, instead of twisted tubules, by a thin ribbon running around its longitudinal axis. The analysis carried out by cryoelectron microscopy (Fig. 3) not only confirms the ribbon nature of the PHFs but also their thickness. A new result obtained when analyzing the height profiles of the ribbons is the repetitive appearance of a small cleft in the lateral profile (Fig. 2E). This could suggest that the ribbon is composed of two strands.

The simulation of the results obtained by AFM with the two types of tips and three different models (Fig. 4) shows that the standard tip can not differentiate between a one-strand and a twostrand ribbon provided that their total width is similar $(16 \mathrm{~nm})$. The use of the ultra-sharp tip however is capable of discriminating between several models, and the simulation performed with this tip shows that a model made up by two thin strands with a large separation between them (Fig. 4F) is not compatible with the experimental image (Fig. 4A). However, a two-strand 
structure with a small separation $(1 \mathrm{~nm})$ (Fig. 4I) could be compatible with what is experimentally found. The separation has similar dimensions to the valley found in the lateral density profile of the PHFs imaged by the ultra-sharp tip (Fig. 2E). In summary, the simulations performed strengthen the notion of the PHFs having a twisted ribbon structure but cannot confirm nor rule out that this structure contains two strand polymers with a very narrow separation between the strands.

Another interesting feature we have observed is the absence of the banded pattern if images are acquired with the "Supertips". At first sight, this is very surprising, since "Supertips" should give higher resolution and should thus be capable of imaging these fine structures on the PHFs. One possible explanation for this phenomena is related to tip-sample interaction. Indeed the two kinds of tips are chemically different (Olympus tips: $\mathrm{Si}_{3} \mathrm{~N}_{4}$, "Supertips": $\mathrm{SiO}_{2}$ ). Moreover, on a nanometer scale the hidrophilicity depends on the local radius of curvature. Therefore, possibly the Olympus cantilevers are sensitive to local variations of hydophilicity while the "Supertips" are not. The banding pattern observed would the rather correspond to local variations in the interactions rather than to topographical features. More investigations are needed to further clarify this point.

It has been described that tau protein, the component of PHF, has a rod-like shape in the unassembled form. This rod has a length of $56 \mathrm{~nm}$ [16]. However, it has also been suggested that tau is a highly elastic protein that could contract for more than $300 \%$, yielding a more globular and shorter protein [15]. This contracted conformation could be present in PHF-forming tau, since it has been shown that the Alz50 antibody recognizes a conformational epitope where the first 18 residues are located close to the tubulin binding region of the tau molecule [6], which is present near to the C-terminal region [12] [41]. The data obtained by AFM suggests that PHFs are generated by a repeated unit, perpendicular to the longitudinal axis of the helix, of either 16 
$\mathrm{nm} \times 8 \mathrm{~nm}$ if the ribbon is generated by one strand, or $8 \mathrm{~nm} \times 8 \mathrm{~nm}$ if the ribbon is made up by two strands. Based on the height profiles of the PHFs generated with the high resolution tips (Fig. 2E), we can assume that the last case is correct and then there would be as a basic unit building the double strand helix, a "brick" of $8 \mathrm{~nm}$ wide $\times 8 \mathrm{~nm}$ long $\times 3 \mathrm{~nm}$ thick, which accounts for 192 $\mathrm{nm}^{3}$ or $154 \mathrm{kDa}$, if a protein density of $0.8 \mathrm{kDa} / \mathrm{nm}^{3}$ is assumed. Taking into account that tau protein has a relative molecular mass of $40 \mathrm{kDa}$, we can hypothesize that each of the structural units contains four molecules of tau. Indeed, it was found that tau protein, in solution, is able tpo form tetramer-like oligomers [9]. On the other hand, Ruben et al. [37] have proposed that tau molecules can form trimers, but these oligomers could be rather small to form the basic unit building PHF.

A working model like shown in figure 5 could be postulated in which tau oligomers, (presumabily tetramers, although further work should be able to confirm it) are placed in a double helical polymer structure.

Our AFM images show that the structure of the PHFs is, basically, composed by a twisted sheet. This structure, probably, contains two strand polymers with a very narrow separation between the strands. There was detected a repetitive unit of 8-12 nm, perpendicular to the longitudinal axis of the helix. We propose that this basic unit is composed by four molecules of tau, regarding the dimensions, the molecular mass and the density of the protein. All the results we confirmed by cryoelectron microscopy. Finally, a working model, which encloses all the conclusions of the manuscript, is proposed. 


\section{Acknowledgements}

We like to acknowledge Dr. J. L. Carrascosa for critical reading of the manuscript.

This work was supported by a grant of Comunidad Autónoma de Madrid (to A.M.B.) and by grants from CICYT (Spain), Fundación La Caixa and by an institutional grant from Fundación Ramón Areces. FM is a recipient of a fellowship of Comunidad Autónoma de Madrid. 


\section{FIGURE LEGENDS}

Fig. 1. AFM images of PHFs on mica support. AFM images were taken with a regular tip (15 nm radius, $1 \mathrm{~N} / \mathrm{m}, 75 \mathrm{kHz}$ ) operating in non contact tapping mode. Fig. 1A and B are topographic images of PHFs at different magnifications. The imaged structures appear as right-handed filaments as it can clearly be observed. A substructure with a period of $8-12 \mathrm{~nm}$ is clearly observed at high magnification in $(\mathrm{C})$ when the derivative image of the raw data is presented. The 3D representation enforces this subsestructure (Fig. 1D)

Fig. 2. PHFs on mica imaged with SuperSharpSilicon ${ }^{\circledR}$-Tips of $5 \mathrm{~nm}$ tip radius, a nominal force constant of $11 \mathrm{~N} / \mathrm{m}$ and a resonance frequency of $120 \mathrm{kHz}$. Images were recorded at typical scan frequencies of $2 \mathrm{~Hz}$. A) shows a high resolution image of PHFs. A detail of a PHF is shown in B). Profiles done in B) are exhibited in C-E). The analysis of the data shows a longitudinal periodicity of $82 \mathrm{~nm}$; a thickness at half height in the sharp area of the filament of $5.5 \mathrm{~nm}$ and a width in the flat area of $17.5 \mathrm{~nm}$. The contribution of the size tip in these values is minimized compared with the values obtained with the Olympus type cantilevers: 82,31 and $30 \mathrm{~nm}$ respectively (data not shown). The measured height of the structure in the sharp area is $15 \mathrm{~nm}$ and $7 \mathrm{~nm}$ in the flat area.

Fig. 3. Cryoelectron microscopy of PHFs. The helicoidal structures depicted here seem to be generated by a thin ribbon turning around is longitudinal axis. In some of them (see the arrow in A), a substructure perpendicular to the direction of the helix is clearly visible. Bar represents 60 nm.

Fig. 4. AFM simulation. Dilation simulation of the models proposed, using the two types of cantilever employed. The simulated images can be compared with the experimental ones (A and 
B) obtained with the ultra-sharp and the standard tip, respectively. Model 1 is a twisted ribbon of $16 \mathrm{~nm}(\mathrm{C}) . \mathrm{D}$ and $\mathrm{E}$ are the simulated images using the tips shown in the insets. Model 2 consists of two paired strands of $4 \mathrm{~nm}$ separated by $9 \mathrm{~nm}(\mathrm{~F})$ and the simulated images for the two tips are $\mathrm{G}$ and $\mathrm{H}$. Model 2 consists of two paired strands of $8 \mathrm{~nm}$ separated by $1 \mathrm{~nm}$ (I) and the simulated images for the two tips are $\mathrm{J}$ and $\mathrm{K}$. Bars indicate A) and B) $43 \mathrm{~nm}$.

Figure 5. Structure model of the PHF polymers. A two-strand polymer with a very narrow separation between the strands could generate the structure of the PHFs (A). The basic structure $(8 \mathrm{~nm} \times 8 \mathrm{~nm} \times 3 \mathrm{~nm})$ would be composed of tetramers of tau protein (B). 


\section{REFERENCES}

[1] D.M. Appelt and B.J. Balin, Analysis of paired helical filaments (PHFs) found in Alzheimer's disease using freeze-drying/rotary shadowing, J. Struct. Biol., 111 (1993) 85-95.

[2] P.V. Arriagada, J.H. Growdon, E.T. Hedley-Whyte and B.T. Hyman, Neurofibrillary tangles but not senile plaques parallel duration and severity of Alzheimer's disease, Neurology, 42 (1992) 631-639.

[3] G. Binnig, C.F. Quate and C. Gerber, Atomic Force Microscope, Physical Review Letters, 56 (1986) 930-933.

[4] J.P. Brion, H. Passasiro, J. Nuñez and J. Flament-Durand, Mise en evidence immunologique de la proteine tau an niveau des lesions degenerescence neurofibrillaire de la maladie d'Alzheimer, Arch. Biol., 95 (1985) 229-235.

[5] C. Bustamante, C. Rivetti and D.J. Keller, Scanning force microscopy under aqueous solutions, Curr. Opin. Struct. Biol., 7 (1997) 709-16.

[6] G. Carmel, E.M. Mager, L.I. Binder and J. Kuret, The structural basis of monoclonal antibody Alz50's selectivity for Alzheimer's disease pathology, J. Biol. Chem., 271 (1996) 3278932795 .

[7] R.A. Crowther, O.F. Olesen, M.J. Smith, R. Jakes and M. Goedert, Assembly of Alzheimer-like filaments from full-length tau protein, FEBS Lett., 337 (1994) 135-138.

[8] A. Engel, C.A. Schoenenberger and D.J. Muller, High resolution imaging of native biological sample surfaces using scanning probe microscopy, Curr. Opin. Struct. Biol., 7 (1997) 279-84. 
[9] J. García de Ancos, I. Correas and J. Avila, Differences in microtubule binding and selfassociation abilities of bovine brain tau isoforms., J. Biol. Chem., 268 (1993) 7976-7982.

[10] M. Goedert, Filamentous nerve cell inclusions in neurodegenerative diseases: tauopathies and alpha-synucleinopathies, Philos. Trans. R. Soc. Lond. B. Biol. Sci, 354 (1999) 1101-1118.

[11] M. Goedert, R. Jakes and R.A. Crowther, Effects of frontotemporal dementia FTDP-17 mutations on heparin-induced assembly of tau filaments, FEBS Lett, 450 (1999) 306-311.

[12] M. Goedert, C.M. Wischik, R.A. Crowther, J.E. Walker and A. Klug, Cloning and sequencing of the cDNA encoding a core protein of the paired helical filament of Alzheimer disease: identification as the microtubule-associated protein tau, Proc. Natl. Acad. Sci. USA, 85 (1988) 4051-4055.

[13] S.G. Greenberg and P. Davies, A preparation of Alzheimer paired helical filaments that displays distinct tau proteins by polyacrylamide gel electrophoresis, Proc. Natl. Acad. Sci. USA, 87 (1990) 5827-31.

[14] I. Grundke-Iqbal, K. Iqbal, Y.C. Tung, M. Quinlan, H.M. Wisniewski and L.I. Binder, Abnormal phosphorylation of the microtubule-associated protein tau (tau) in Alzheimer cytoskeletal pathology, Proc. Natl. Acad. Sci. USA, 83 (1986) 4913-4917.

[15] T. Hagestedt, B. Lichtenberg, H. Wille, E.M. Mandelkow and E. Mandelkow, Tau protein becomes long and stiff upon phosphorylation: correlation between paracrystalline structure and degree of phosphorylation, J. Cell Biol., 109 (1989) 1643-51.

[16] N. Hirokawa, Y. Shiomura and S. Okabe, Tau proteins: the molecular structure and mode of binding on microtubules, J Cell Biol, 107 (1988) 1449-59. 
[17] Y. Ihara, N. Nukina, R. Miura and M. Ogawara, Phosphorylated tau protein is integrated into paired helical filaments in Alzheimer's disease, J. Biochem. Tokyo, 99 (1986) 1807-10.

[18] M.D. Ikonomovic, D.M. Armstrong, S.H. Yen, C. Obcemea and B. Vidic, Atomic force microscopy of paired helical filaments isolated from the autopsied brains of patients with Alzheimer's disease and immunolabeled against microtubule-associated protein tau, American Journal of Pathology, 147 (1995) 516-528.

[19] M. Kidd, Paired helical filaments in electron microscopy of Alzheimer's disease, Nature, 197 (1963) 192-193.

[20] K.S. Kosik, S. Bakalis, L. Galibert, D.J. Selkoe and L.K. Duffy, Age-related modifications of MAP-2, Ann N Y Acad Sci, 466 (1986) 420-2.

[21] K.S. Kosik, S.F. Bakalis, D.J. Selkoe, M.W. Pierce and L.K. Duffy, High molecular weight microtubule-associated proteins: purification by electro-elution and amino acid compositions, J. Neurosci. Res., 15 (1986) 543-51.

[22] T. Kowalewski and D.M. Holtzman, In situ atomic force microscopy study of Alzheimer's beta-amyloid peptide on different substrates: new insights into mechanism of beta- sheet formation, Proc. Natl. Acad. Sci. USA, 96 (1999) 3688-3693.

[23] E. Margeat, C. Le Grimellec and C.A. Royer, Visualization of trp repressor and its complexes with DNA by atomic force microscopy, Biophys J, 75 (1998) 2712-20.

[24] E. Montejo de Garcini, J.L. Carrascosa, I. Correas, A. Nieto and J. Avila, Tau factor polymers are similar to paired helical filaments of Alzheimer's disease, FEBS Lett., 236 (1988) $150-154$. 
[25] E. Montejo de Garcini, L. Serrano and J. Avila, Self assembly of microtubule associated protein tau into filaments resembling those found in Alzheimer disease., Biochem. Biophys. Res. Commun., 141 (1986) 790-796.

[26] F. Moreno-Herrero, P.J. de Pablo, J. Colchero, J. Gómez-Herrero and A.M. Baró, The role of shear forces in scanning force microscopy: a comparison between the jumping mode and tapping mode, Surf. Sci., 453 (2000) 152-158.

[27] F. Moreno-Herrero, P. Herrero, J. Colchero, A.M. Baro and F. Moreno, Analysis by atomic force microscopy of Med8 binding to cis-acting regulatory elements of the SUC2 and HXK2 genes of saccharomyces cerevisiae, FEBS Lett., 459 (1999) 427-432.

[28] F. Moreno-Herrero, P. Herrero, J. Colchero, A.M. Baro and F. Moreno, Imaging and mapping protein-binding sites on DNA regulatory regions with atomic force microscopy, Biochem. Biophys. Res. Commun., 280 (2001) 151-157.

[29] B. Muller-Hill and K. Beyreuther, Molecular biology of Alzheimer's disease, Annu. Rev. Biochem., 58 (1989) 287-307.

[30] A. Nieto, I. Correas, E. Montejo de Garcini and J. Avila, A modified form of microtubuleassociated tau protein is the main component of paired helical filaments., Biochem. Biophys. Res. Commun., 154 (1988) 660-667.

[31] K. Ohtsubo, N. Izumiyama, H. Shimada, T. Tachikawa and H. Nakamura, Threedimensional structure of Alzheimer's neurofibrillary tangles of the aged human brain revealed by the quick-freeze, deep-etch and replica method, Acta Neuropathol. Berl., 79 (1990) 480-485. 
[32] M. Pérez, J.M. Valpuesta, M. Medina, E. Montejo de Garcini and J. Avila, Polymerization of tau into filaments in the presence of heparin: the minimal sequence required for tau-tau interaction., J. Neurochem., 67 (1996) 1183-1190.

[33] M.S. Pollanen, P. Markiewicz, C. Bergeron and M.C. Goh, Twisted ribbon structure of paired helical filaments revealed by atomic force microscopy, Am. J. Pathol., 144 (1994) 869-873.

[34] M.S. Pollanen, P. Markiewicz and M.C. Goh, Paired helical filaments are twisted ribbons composed of two parallel and aligned components: Image reconstruction and modeling of filament structure using atomic force microscopy, J Neuropathol Exp Neurol, 56 (1997) 79-85.

[35] M.S. Pollanen, P. Markiewicz, M.C. Goh and C. Bergeron, Alzheimer paired helical filaments: A comparison with the twisted ribbon model, Acta Neuropathologica, 90 (1995) 194197.

[36] G.C. Ruben, K. Iqbal, I.I. Grundke and J.J. Johnson, The organization of the microtubule associated protein tau in Alzheimer paired helical filaments, Brain Res, 602 (1993) 1-13.

[37] G.C. Ruben, K. Iqbal, I.I. Grundke, H.M. Wisniewski, T.L. Ciardelli and J.J. Johnson, The microtubule-associated protein tau forms a triple-stranded left-hand helical polymer, J Biol Chem, 266 (1991) 22019-27.

[38] G.C. Ruben, K. Iqbal, H.M. Wisniewski, J.J. Johnson and I.I. Grundke, Alzheimer neurofibrillary tangles contain $2.1 \mathrm{~nm}$ filaments structurally identical to the microtubule-associated protein tau: a high-resolution transmission electron microscope study of tangles and senile plaque core amyloid, Brain Res., 590 (1992) 164-79. 
[39] G.C. Ruben, J.Z. Wang, I. GrundkeIqbal and K. Iqbal, Paired helical filaments have a wide range of widths with similar helical periods. In K. Iqbal, D.F. Swaab, B. Winblad and H.M. Wisniewski (Eds.), Alzheimer's Disease and Relat, John Wiley \& Sons Ltd, Baffins Lane, Chichester PO19 1UD, West Sussex, England, 1999, pp. 187-192.

[40] Z. Shao, J. Mou, D.M. Czajkowsky, J. Yang and Y. Yuan, Biological atomic force microscopy: what is achieved and what is needed, Adv. Phys., 45 (1996) 1-86.

[41] A. Takeda, M.A. Smith, J. Avila, A. Nunomura, S.L. Siedlak, X. Zhu, G. Perry and L.M. Sayre, In Alzheimer's disease, heme oxygenase is coincident with Alz50, an epitope of tau induced by 4-hydroxy-2-nonenal modification, J. Neurochem., 75 (2000) 1234-41.

[42] R.D. Terry and H. Wisniewski, M,, Ultrastructure of senile dementia and of experimental analogs. In C.M. Gaitz (Ed.), Aging and brain, Plenum Publishing Corporation, New York, 1972, pp. 89-116.

[43] S.J. van Noort, K.O. van der Werf, A.P. Eker, C. Wyman, B.G. de Grooth, N.F. van Hulst and J. Greve, Direct visualization of dynamic protein-DNA interactions with a dedicated atomic force microscope, Biophys. J., 74 (1998) 2840-9.

[44] J.S. Villarubia, Scanned probe microscope tip characterization without calibrated characterizers, J. Vac. Sci. Technol., B14(2) (1996) 1518-1521.

[45] H. Wille, G. Drewes, J. Biernat, E.M. Mandelkow and E. Mandelkow, Alzheimer-like paired helical filaments and antiparallel dimers formed from microtubule-associated protein tau in vitro, J. Cell. Biol., 118 (1992) 573-584. 
[46] C.M. Wischik and R.A. Crowther, Subunit structure of the Alzheimer tangle, Br. Med. Bull., 42 (1986) 51-56.

[47] C.M. Wischik, R.A. Crowther, M. Stewart and M. Roth, Subunit structure of paired helical filaments in Alzheimer's disease, J. Cell. Biol., 100 (1985) 1905-1912.

[48] C.M. Wischik, R.Y.K. Lai and C.R. Harrington, Modelling prion-like processing of tau protein in Alzheimer's disease for pharmaceutical development. In J. Avila, R. Brandt and K.S. Kosik (Eds.), Brain Microtubule Associated, Harwood Academic Publ GmbH, Chur, Switzerland, 1997, pp. 185-241.

[49] C.M. Wischik, M. Novak, P.C. Edwards, A. Klug, W. Tichelaar and R.A. Crowther, Structural characterization of the core of the paired helical filament of Alzheimer disease, Proc. Natl. Acad. Sci. USA, 85 (1988) 4884-8.

[50] C.M. Wischik, M. Novak, H.C. Thogersen, P.C. Edwards, M.J. Runswick, R. Jakes, J.E. Walker, C. Milstein, M. Roth and A. Klug, Isolation of a fragment of tau derived from the core of the paired helical filament of Alzheimer disease, Proc. Natl. Acad. Sci. USA, 85 (1988) 45064510.

[51] H. Wisniewski, M, H.K. Narang and R.D. Terry, Neurofibrillary tangles of paired helical filaments, J. Neurol. Sci., 27 (1976) 173-181.

[52] J.G. Wood, S.S. Mirra, N.J. Pollock and L.I. Binder, Neurofibrillary tangles of Alzheimer disease share antigenic determinants with the axonal microtubule-associated protein tau (tau), Proc. Natl. Acad. Sci. USA, 83 (1986) 4040-3. 


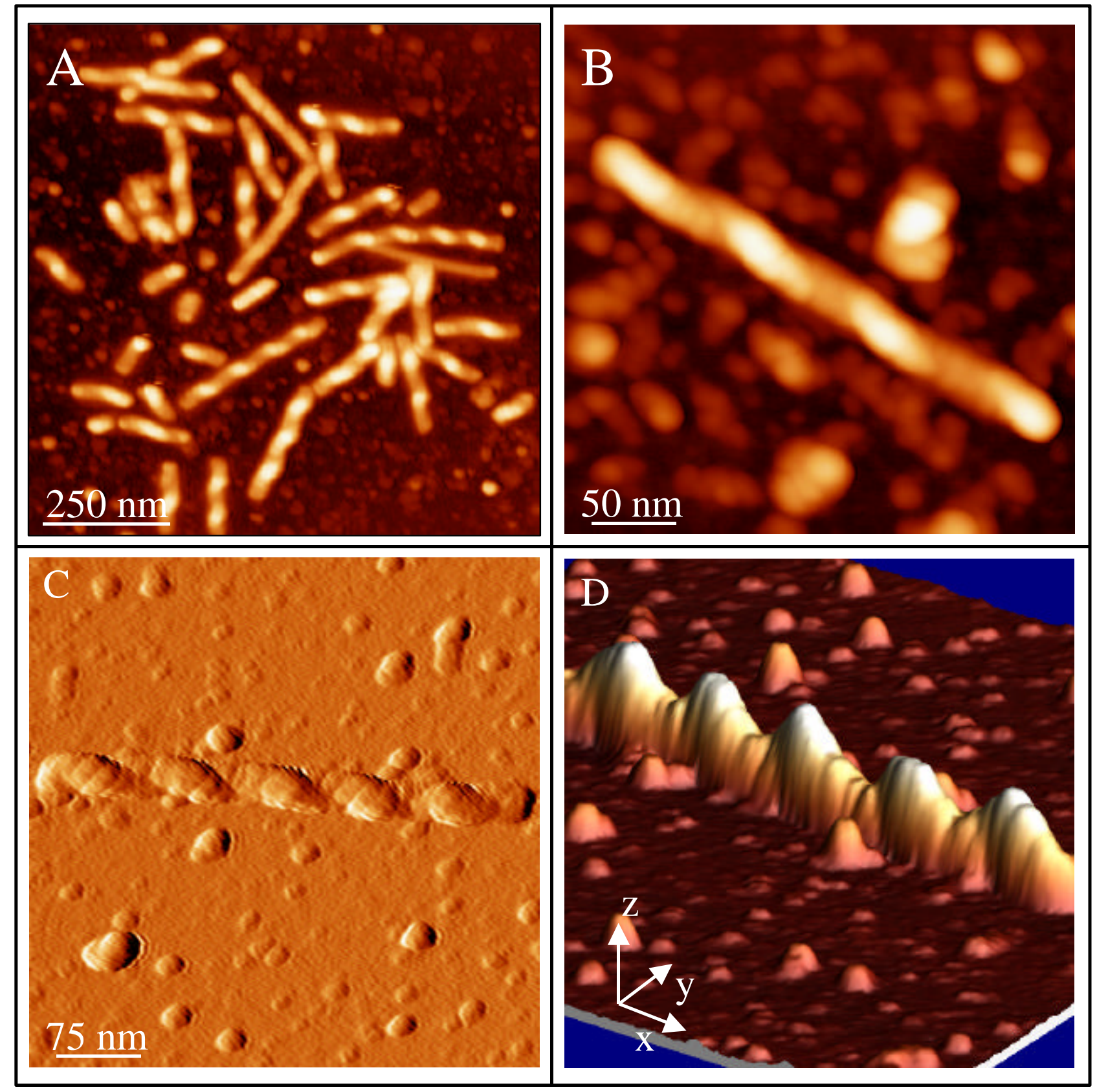

Figure 1 


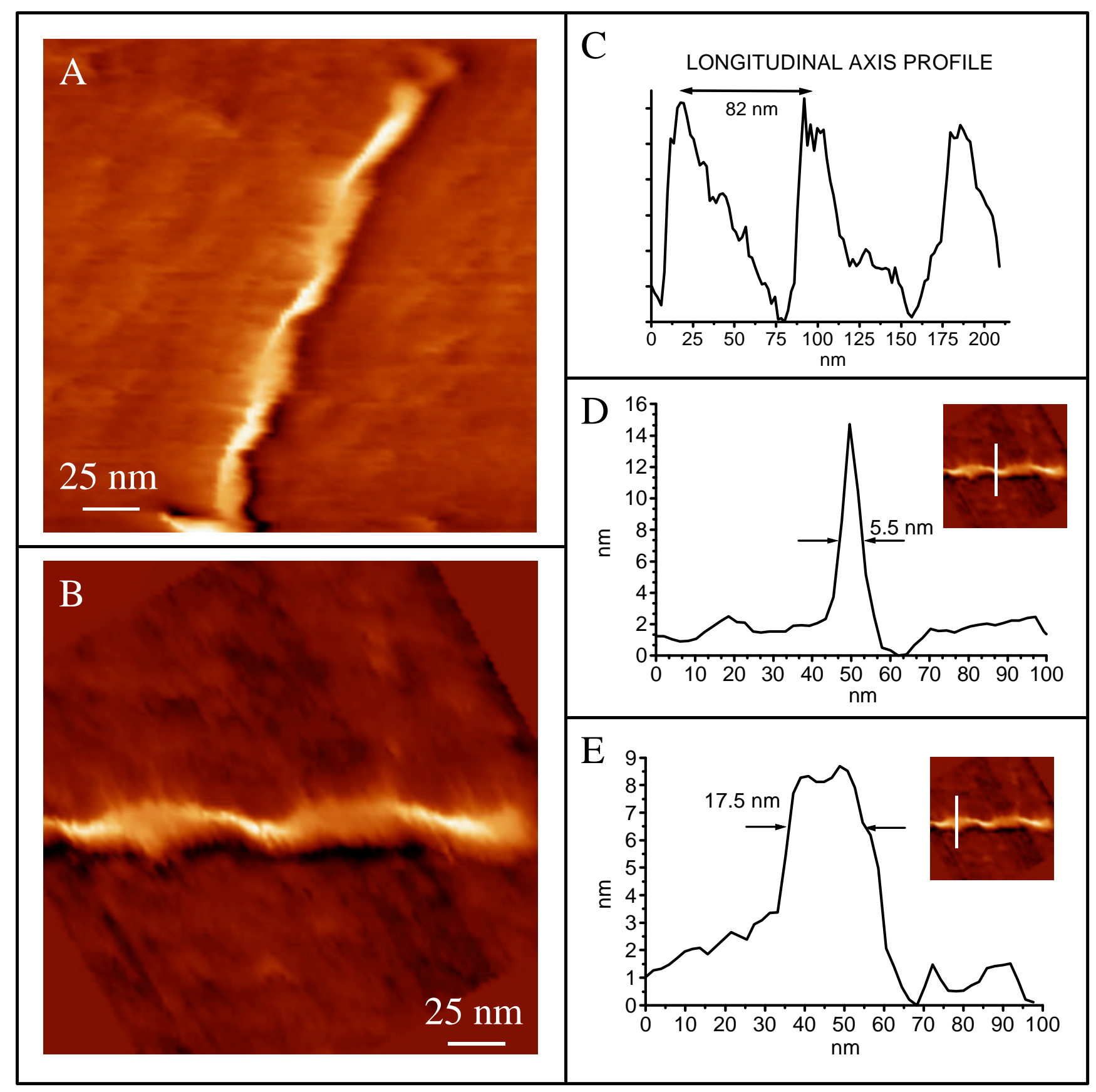

Figure 2 


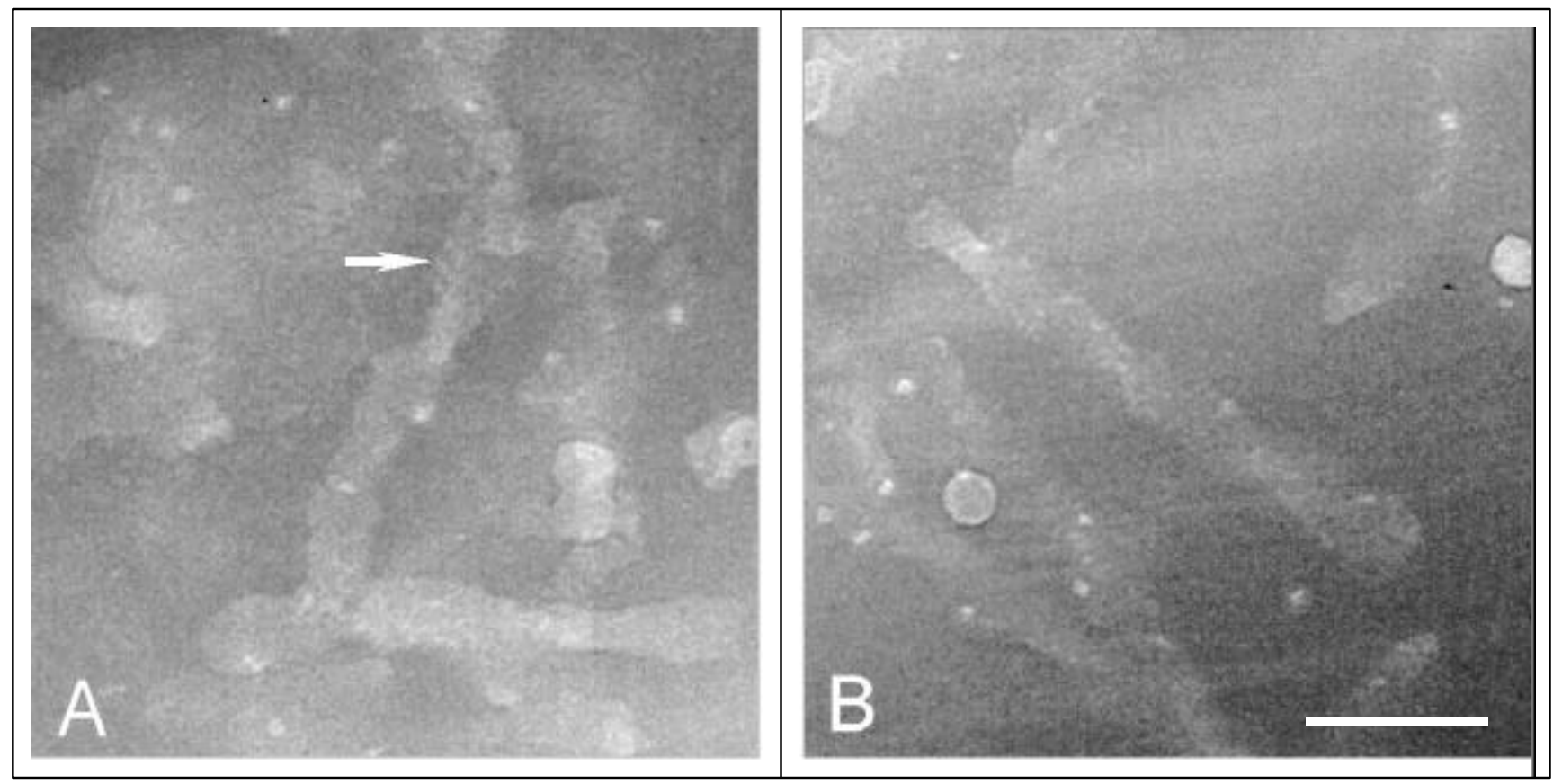

Figure 3 


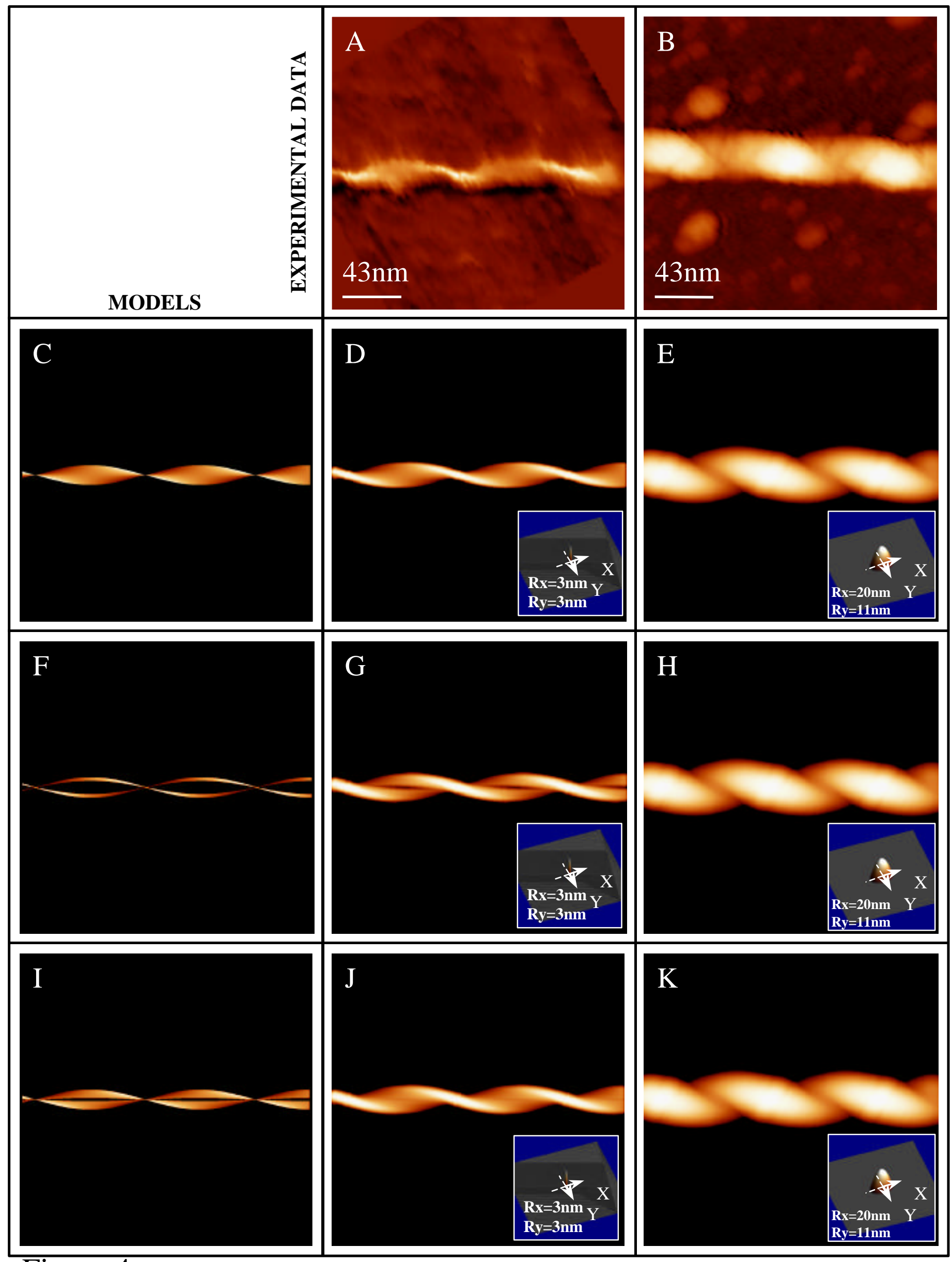

Figure 4 


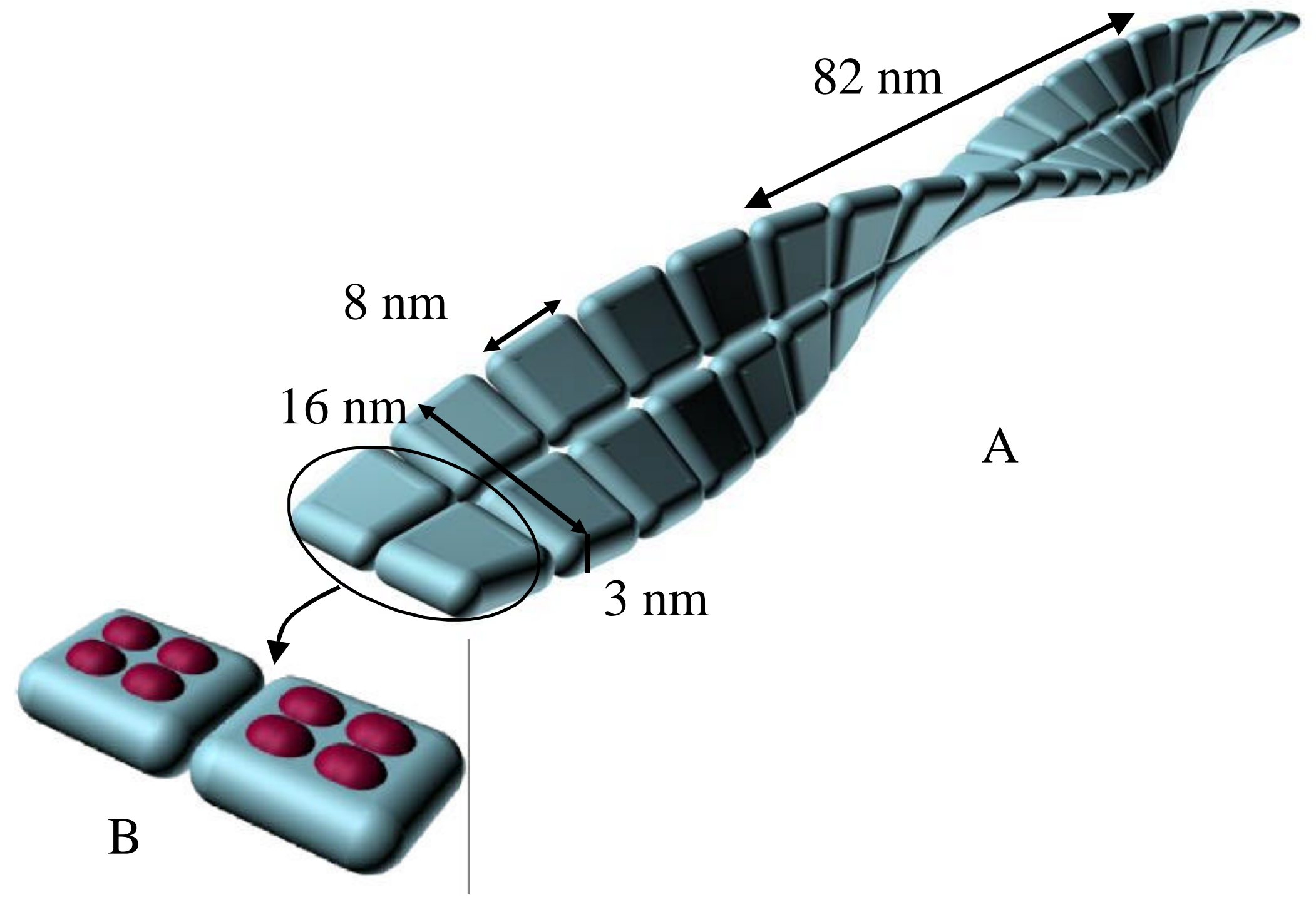

Figure 5 